\title{
QDs Supported on Langmuir-Blodgett Films of Polymers and Gemini Surfactant
}

\author{
T. Alejo, B. Martín-García, M. D. Merchán, and M. M. Velázquez \\ Departamento de Química Física, Facultad de Ciencias Químicas, Universidad de Salamanca, \\ Plaza de los Caídos s/n, 37008 Salamanca, Spain
}

Correspondence should be addressed to M. M. Velázquez; mvsal@usal.es

Received 7 May 2013; Revised 18 June 2013; Accepted 19 June 2013

Academic Editor: Amir Kajbafvala

Copyright (C) 2013 T. Alejo et al. This is an open access article distributed under the Creative Commons Attribution License, which permits unrestricted use, distribution, and reproduction in any medium, provided the original work is properly cited.

Different LB films of poly(octadecene-co-maleic anhydride), PMAO, poly(styrene-co-maleic anhydride) partial 2 butoxy ethyl ester cumene terminated, PS-MA-BEE, and Gemini surfactant ethyl-bis(dimethyl octadecylammonium bromide), 18-2-18, have been used to study the effect of the substrate coating on the surface self-assembly of CdSe quantum dots (QDs). Results show that all the "coating molecules" avoid the 3D aggregation of QDs observed when these nanoparticles are directly deposited on mica. Different morphologies were observed depending on the molecules used as coatings, and this was related to the surface properties, such as wetting ability, and the morphology of the coating LB films.

\section{Introduction}

Nanoparticles have received great interest due to their unique optical, mechanical, and electric properties. For several technological applications it is necessary to support them on a substrate [1]. In these situations, the organization of nanoparticles into ordered arrays can lead to new interesting properties, which are strongly affected by the morphology of the nanoparticle film [2-6]. Nanoparticles tend to agglomerate by attractive interactions $[7,8]$ decreasing the quality of the devices. Therefore, the most recent efforts involve the use of polymer or surfactant molecules to minimize nanoparticles $3 \mathrm{D}$ aggregation. Despite the great interest generated in recent years on this topic, more work must be carried out to develop multifunctional materials with novel electric, magnetic, or optical properties [9].

Several lithographic techniques have been employed to generate well-ordered nanostructures [10-12]; however, these methods are often limited because they require specialized equipment. Layer by layer deposition (LbL) is an alternative approach to self-assembly polymeric nanocomposites [13, 14]. In the LbL methodology the multilayer is achieved by consecutive adsorption of polyelectrolytes and nanoparticles by electrostatic interactions. Consequently, this approach can be only used for charged polymers and nanoparticles, which exclude a wide number of functional materials. LangmuirBlodgett (LB) methodology, based on the transfer process of Langmuir films from the air-water interface onto solids, has proved to be a versatile and interesting tool to obtain water-insoluble thin films. This deposition technique allows the continuous variation of particle density, spacing, and arrangement by compressing or expanding the film using barriers. Consequently, it offers the possibility of preparing reproducible films of polymers, surfactants, and nanoparticles with the control of interparticle distance necessary to exploit the nanocomposites in technological applications [15, 16].

One of the issues of LB methodology lies on the dewetting effects observed when nanoparticles are deposited onto solids $[17,18]$. These dewetting processes induce nanoparticles aggregates that in several circumstances ruin the physical properties of the nanoparticles [19]. To avoid the dewetting process, a widely used strategy consists in modifying the surface properties of solids by covering the substrates with self-assembly monolayers [20-24]. This strategy facilitates the incorporation of nanoparticles onto solids by improving the nanoparticle wettability and has been used to deposit CdSe QDs onto silanes [22-24] and gold nanoparticles onto acrylic block copolymers derivatives [21]. Despite the great interest expressed for this technique, several efforts must be 
made to know the role of coatings on the nanoparticle selfassembly at the interface in order to improve the properties of the nanoparticle films. With this objective in mind, we carried out a systematic study of the effect of the coating molecules on the QDs self-assembly process of CdSe QDs. We have selected three amphiphilic molecules of distinct nature, the copolymers, PMAO and PS-MA-BEE, and the Gemini surfactant 18-2-18. All these molecules present surface activity; therefore, we expect that they anchor to the substrate mica, through their hydrophilic moieties favoring the QDs adhesion across its hydrophobic part. In addition, we chose the polymer PMAO because it is well established that PMAO interacts effectively with hydrophobic nanoparticles leading to excellent stability by avoiding 3D aggregation [2528]. On the other hand, PMAO-capped nanoparticles have been successfully incorporated onto solid substrates by the LB technique [29], and PMAO thin films were employed to make QDs compatible with biosensing applications [30]. Moreover, styrene copolymers, such as PS-MA-BEE, were proposed as good candidates to organize hybrid materials at nanometer scale [31-33] to fabricate submicrometric electronic devices because they present mechanical rigidity and good adhesion on solids $[34,35]$.

On the other hand, surfactants are often used to control nanomaterials self-assembly [36-38]. In particular, Gemini surfactants made of two surfactant monomers linked by a spacer chain display high adsorption on solid surfaces and biological compatibility $[39,40]$. Therefore, LB films of Gemini surfactants such as 18-4-18 and 18-12-18 have been used as substrate coating to improve the adsorption of collagen on solids [20], and some researchers have proposed combining this with DNA for biotechnological applications [41, 42]. Accordingly, we expect that the LB films of the Gemini surfactant 18-2-18 result in good substrate coating for QDs deposition.

The methodology employed in this work consists in transferring nanoparticles of QDs from the air-water interface onto mica previously coated by LB films of PMAO, PSMA-BEE, or the Gemini surfactant 18-2-18. Since several polymer/surfactant mixtures provide synergistic effects on the surface adsorption [43-46], we also studied the ability of mixed PMAO/18-2-18 LB films to modulate the self-assembly of QDs films. The LB film morphology was analyzed by different imaging microcopy techniques, atomic force microscopy (AFM), scanning electron microscopy (SEM), and transmission electron microscopy (TEM).

\section{Materials and Methods}

2.1. Materials. The polymers poly(maleic anhydride-alt-1octadecene), PMAO $(\mathrm{Mr}=40 \mathrm{kDa})$ and poly(styrene-comaleic anhydride) partial 2 butoxy ethyl ester cumene terminated, PS-MA-BEE, were purchased from Sigma-Aldrich. PSMA-BEE with an ester : acid ratio 1:1 and molecular weight $\mathrm{Mr}=2.5 \mathrm{kDa}$ were provided by the manufacturer. The Gemini surfactant ethyl-bis(dimethyl octadecylammonium bromide), 18-2-18, was synthesized using the method described by Zana et al. [47] with some significant modifications in the crystallization step introduced by our group [48] to guarantee the degree of purity $(>99.9 \%)$ necessary for the correct interpretation of surface experiments.

The synthesis of CdSe QDs capped with trioctylphosphine oxide (TOPO) was carried out by the method proposed by $\mathrm{Yu}$ and Peng [49], described in more detail in [19]. The QDs size $(3.55 \pm 0.05 \mathrm{~nm})$ was determined by the position of the maximum of the visible spectrum of the QDs dispersed in chloroform [50]. We calculated the concentration of nanocrystals from the UV-Vis absorption spectrum of QDs dissolved in chloroform by using the extinction coefficient per mole of nanocrystals at the first excitonic absorption peak [50]. UV-Vis absorption spectra of QDs solutions were recorded on a Shimadzu UV-2401PC spectrometer.

Chloroform (PAI, filtered) used to prepare the spreading solutions was purchased from Sigma-Aldrich. Millipore ultrapure water prepared using a combination of RiOs and Milli-Q systems was used as subphase. The LB substrate muscovite (mica) quality V-1 was supplied by EMS (USA) and was freshly cleaved before use.

2.2. Preparation of Langmuir Monolayers and LangmuirBlodgett Films. The surface pressure measurements were performed on a computer-controlled Teflon Langmuir film balance Standard KSV2000-2 (KSV, Finland). Spreading solution was deposited onto water subphase with a Hamilton microsyringe. The syringe precision is $1 \mu \mathrm{L}$. The surface pressure was measured with a Pt-Wilhelmy plate connected to an electrobalance. The subphase temperature was maintained at $(20.0 \pm 0.1)^{\circ} \mathrm{C}$ by flowing thermostated water through jackets at the bottom of the trough. The temperature near the surface was measured with a calibrated sensor from KSV. The water temperature was controlled by means of a Lauda Ecoline RE-106 thermostat/cryostat. Langmuir monolayers were transferred by symmetric barrier compression $\left(5 \mathrm{~mm} \mathrm{~min}^{-1}\right)$ by the vertical dipping method at a rate of $5 \mathrm{~mm} \mathrm{~min}^{-1}$, forming Y-type LB films. The methodology employed for the bilayer preparation is as follows. First, the polymer or surfactant film is LB deposited. After deposition, the film is dried under vacuum, and then, the QD layer is transferred onto the surfactant or polymer LB film by the LB methodology. To differentiate between the components and deposition order of the different LB films, we will employ the notation // to separate the components of the two layers in contact, and the components order is from solid substrate to air.

2.3. Scanning Electron Microscopy (SEM). A FEI Helios 450 dual-beam FIB/SEM was used to obtain images of the LB films with a supply to the electron beam between 5 and $10 \mathrm{kV}$.

2.4. Atomic Force Microscopy (AFM). AFM images of the LB films deposited on mica were obtained in constant repulsive force mode by AFM (Nanotec Dulcinea, Spain) with a rectangular microfabricated silicon nitride cantilever (Olympus OMCL-RC800PSA) with a height of $100 \mu \mathrm{m}$, a Si pyramidal tip (radius $<20 \mathrm{~nm}$ ), and a spring constant of $0.73 \mathrm{~N} \mathrm{~m}^{-1}$. The scanning frequencies were usually in the range between 0.5 and $2.0 \mathrm{~Hz}$ per line. The measurements were carried out under ambient laboratory conditions. 
2.5. Transmission Electron Microscopy (TEM). TEM images of the LB films deposited on Formvar-carbon-coated copper grids were taken with $80 \mathrm{kV}$ TEM (ZEISS EM 902, Germany). The LB deposition onto copper grids was realized at a speed up to $1 \mathrm{~mm} \mathrm{~min}^{-1}$.

\section{Results}

To evaluate the effect of the polymer hydrophobic block on the morphology of QDs films, we used two maleic anhydride polymer derivatives containing hydrocarbon chains (PMAO) or styrene moieties (PS-MA-BEE) as hydrophobic blocks.

To optimize the QDs deposition, it is necessary to characterize the polymer Langmuir monolayers precursors of the coating LB films. In previous work, we have studied the surface properties of these polymers monolayers [45,53]. Our results showed that the PS-MA-BEE isotherm is characteristic of block copolymers with a plateau at $\sim 30 \mathrm{mN} \mathrm{m}^{-1}$ attributed to the onset of polymer phase transition towards brush conformation, while the PMAO isotherm is typical of an amphiphilic material in which the collapse is reached at the surface pressure value of $40 \mathrm{mN} \mathrm{m}^{-1}$ [45]. The equilibrium elasticity values, calculated from the isotherms and $\varepsilon_{0}=$ $\Gamma(\delta \pi / \delta \Gamma)_{T}$, allowed us to obtain information of the surface states, and significant differences were observed between the polymer films. Thus, the PMAO monolayer is more elastic than PS-MA-BEE and reaches the maximum $\varepsilon_{0}$ value of $167 \mathrm{mN} \mathrm{m}^{-1}$ at a surface pressure of $30 \mathrm{mN} \mathrm{m}^{-1}$ [45], while the maximum elasticity value found for monolayers of PSMA-BEE was $60 \mathrm{mN} \mathrm{m}^{-1}$ at $\pi=14 \mathrm{mN} \mathrm{m}^{-1}$ [54].

To prepare the QDs films using this methodology, the first step is to obtain dense LB films of polymers by transferring the densest Langmuir monolayers of monomers avoiding polymer aggregation. According to this procedure, we transferred Langmuir monolayers at the maximum elasticity value because beyond this value the monolayer collapses, resulting in $3 \mathrm{D}$ polymer aggregates at the interface. These $3 \mathrm{D}$ aggregates are metastable and lead to nonreproducible results, which are not desirable. Therefore, the LB films were prepared by transferring Langmuir monolayers of PS-MA-BEE at $14 \mathrm{mN} \mathrm{m}^{-1}$ and of PMAO at $30 \mathrm{mN} \mathrm{m}^{-1}$, respectively. The AFM images of these polymer films were obtained in previous work and showed that the PS-MA-BEE film presents wide stripes along the film [53] with height profile values ranging from 8 to $10 \mathrm{~nm}$, while the PMAO LB film presents some small holes produced by dewetting processes [45]. The next step was to deposit QDs onto LB films of these polymers. The surface pressure value of the QD monolayer transferred on the substrate coating was $9 \mathrm{mN} \mathrm{m}^{-1}$.

To evaluate the film morphology at different scales, we used AFM, TEM, and SEM. Representative images of different films are collected in Figure 1. From these images one can see different morphologies depending on the polymer used as coating. Figures 1(a) and 1(b) show the AFM images of QDs deposited onto PS-MA-BEE and PMAO, respectively. Significant differences can be seen. Thus, the QDs films prepared on PS-MA-BEE are constituted by island-like aggregates of different sizes (Figure 1(a)). The TEM image of this film (Figure 1(c)), allows analysis of the island architecture, demonstrating high polydispersity degree in the island size. Conversely, the QDs film deposited onto PMAO LB film (Figure 1(b)) presents higher coverage than that deposited onto PS-MA-BEE (Figure 1(a)). TEM image of PMAO//QD film (Figure 1(d)) presents a magnification of this film and shows isolated nanoparticles deposited along the film. Some regions without nanoparticles are observed in both AFM and TEM images. These regions can be compatible with zones less densely covered observed on the PMAO films used as substrate coating [45]. This fact seems to indicate that the QDs are preferentially adsorbed on PMAO.

The height profiles obtained from the AFM images were also determined, as shown in Figures 1(a) and 1(b), and results demonstrated that the QD film height is independent of the polymer used as coating. The average value calculated from several images, $(3 \pm 1) \mathrm{nm}$, is compatible with the diameter of CdSe nanoparticles. Therefore, results demonstrate that deposition onto LB films of PS-MA-BEE and PMAO avoids the QDs 3D aggregation. These 3D structures were clearly observed when the QDs films are deposited on mica without previous treatment of the substrate [45].

Our results demonstrate that the polymer PMAO favors the QDs deposition compared with the polymer PS-MA-BEE. To interpret this behavior, it is necessary to consider that the polymer PMAO and the QDs ligand (TOPO) present hydrocarbon chains as hydrophobic groups, while the hydrocarbon moiety of the polymer PS-MA-BEE contains styrene groups. Accordingly, the poly-styrene groups likely present a more unfavorable interaction with the hydrocarbon chains of TOPO than the hydrocarbon chains of PMAO, and the QDQD interaction prevails, resulting in island-like aggregates.

We explore the ability of the surfactant 18-2-18 to adsorb nanoparticles of QDs. This surfactant molecule contains the same hydrocarbon chain as the polymer PMAO and renders an almost continuous LB film of close-packed surfactant molecules [48]; therefore, it could improve the adsorption of QDs resulting in a more continuous and homogeneous QDs film than PMAO.

The LB surfactant film was built by transferring dense Langmuir monolayers before the collapse, $30 \mathrm{mN} \mathrm{m}^{-1}$. The height profile of the surfactant LB film, (Figures 2(a) and 2(b)), determined by AFM was $2.2 \mathrm{~nm}$. This value agrees with the length of the fully extended hydrocarbon chain of 18 methylene groups $(2.4 \mathrm{~nm})$ [55]. This fact indicates that the surfactant molecules are nearly vertical to the air-solid interface and expose its hydrophobic moiety to the QDs ligand deposited onto the LB surfactant film. We transferred the QDs Langmuir monolayer at the surface pressure value of $9 \mathrm{mN} \mathrm{m}^{-1}$ onto the Gemini surfactant LB film. Figures 2(c) and 2(d) correspond to the SEM and TEM images of QDs films deposited onto the surfactant LB film. The SEM image (Figure 2(c)), shows large domains of QDs while the TEM image allows us to analyze the structure inside domains, as shown in Figure 2(d). In the AFM measurements, the height profile obtained for the QDs domains was 3-4 nm. This value is compatible with the diameter of the nanoparticles, indicating that the surfactant LB film also avoids the $3 \mathrm{D}$ aggregation of nanoparticles. 

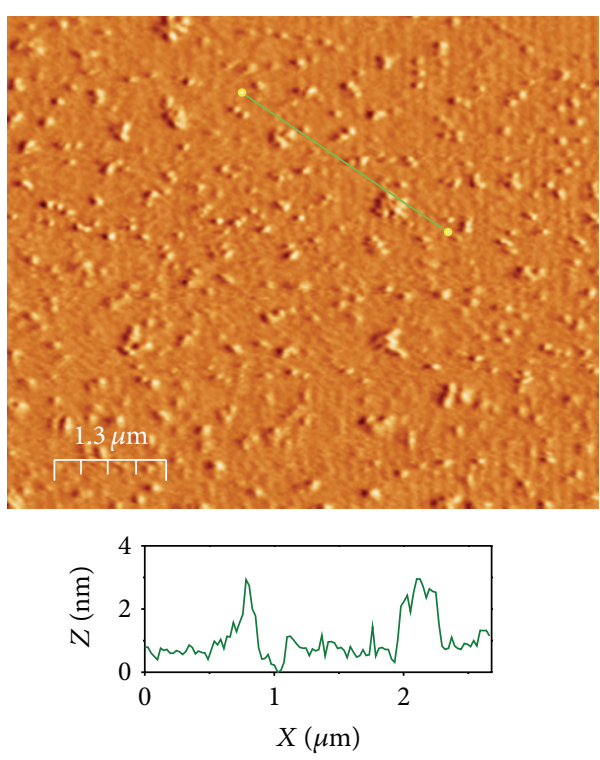

(a)

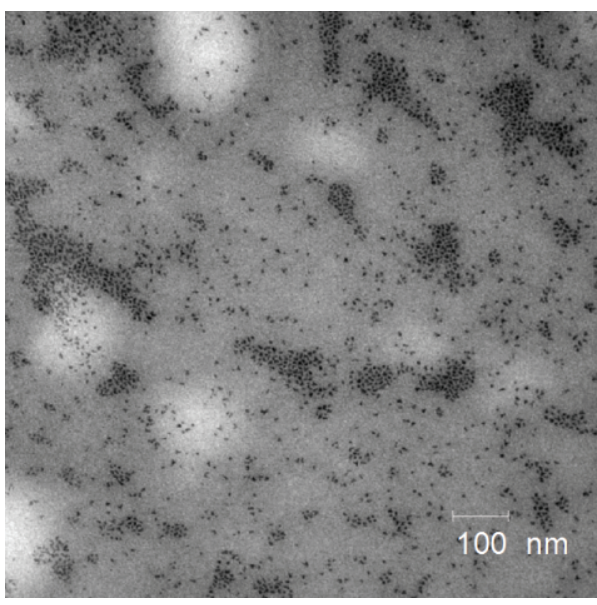

(c)
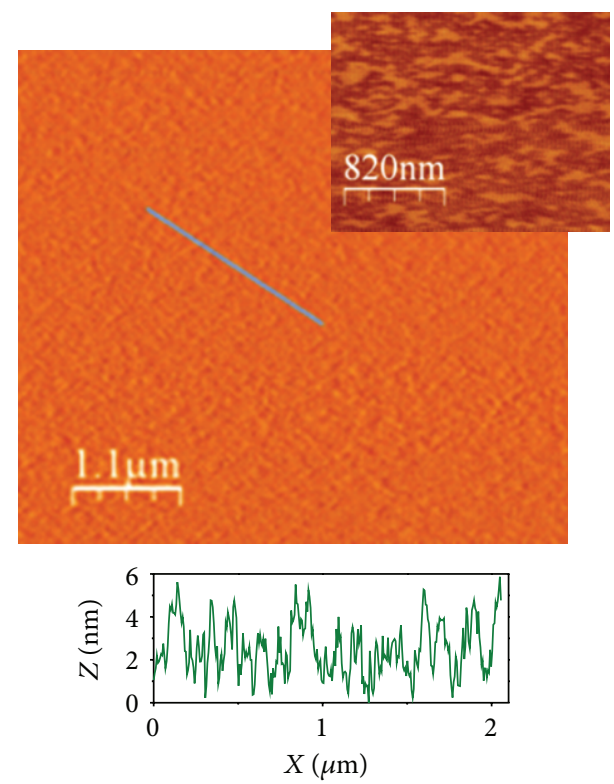

(b)

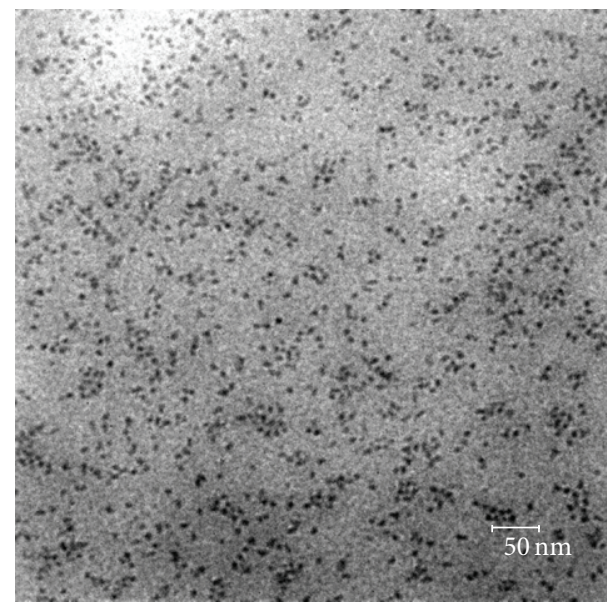

(d)

Figure 1: AFM ((a), (b)) and TEM ((c), (d)) images of PS-MA-BEE//QDs ((a), (c)) and PMAO//QDs ((b), (d)) bilayer LB films. The inset shows a magnification of a PMAO topographic AFM image. The PS-MA-BEE and PMAO Langmuir monolayers were transferred at the surface pressure of 14 and $30 \mathrm{mN} \mathrm{m}^{-1}$, respectively, and QDs at $9 \mathrm{mN} \mathrm{m}^{-1}$.

From the TEM image in Figure 2(d), it can be seen that the QDs domains are constituted by close-packed nanoparticles. To evaluate the distance between QDs inside domains we have calculated the radial distribution function (RDF), $g(r)$, by using the Image 1.46 software [56], as shown in Figure 2(e). The RDF is determined from the distribution of interparticle center distances $(r)$ in several TEM images where the QDs are densely packed. This function provides information about the short-range order inside the QDs domains. The RDF in Figure 2(e) represents the normalized function referred to the maximum value showing a distribution centered at $r=5.1 \pm 0.1 \mathrm{~nm}$. From this value, the interparticle distance $(d)$ was calculated, and the value found was $1.7 \mathrm{~nm}$. This distance is slightly higher than two times the length of the TOPO coating molecules $(0.7 \mathrm{~nm})$ [57] and is consistent with a close-packed QDs arrangement. Summarizing, our results demonstrate that the most packed QDs domains are obtained for films built by transferring QDs Langmuir monolayers from the air-water interface onto the Gemini surfactant 18-2-18 LB film. Moreover, results show the importance of both the attractive interactions between the QDs ligand (TOPO) and the molecules used to modify the properties of the solid support and the morphology of the coating film.

In an attempt to interpret this behavior, we analyzed the ability of QDs to wet the different molecules employed as substrate coating by means of the spreading parameter. The spreading parameter has been used previously in LB 


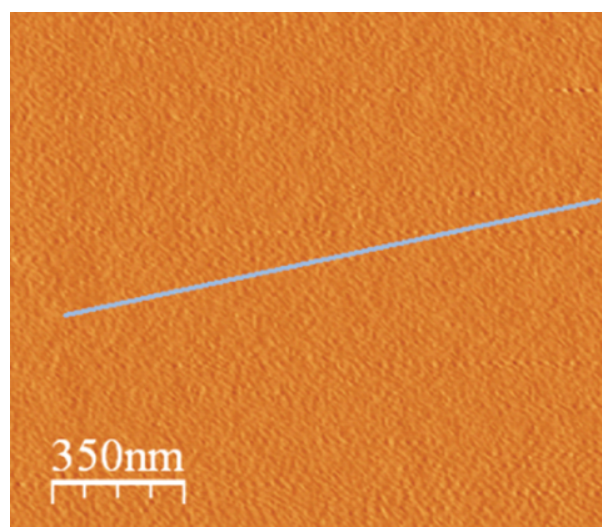

(a)

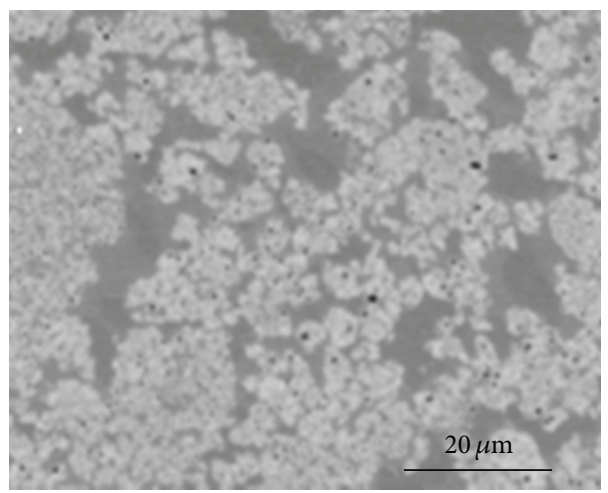

(c)
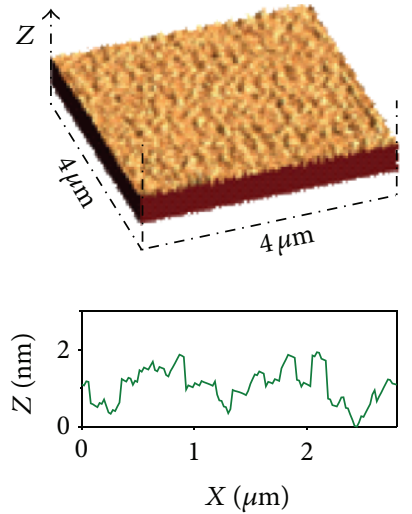

(b)

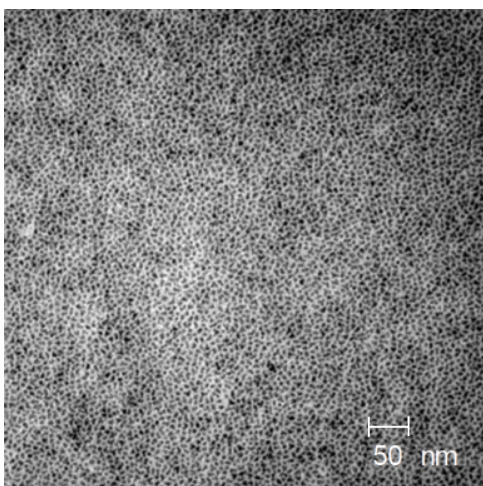

(d)

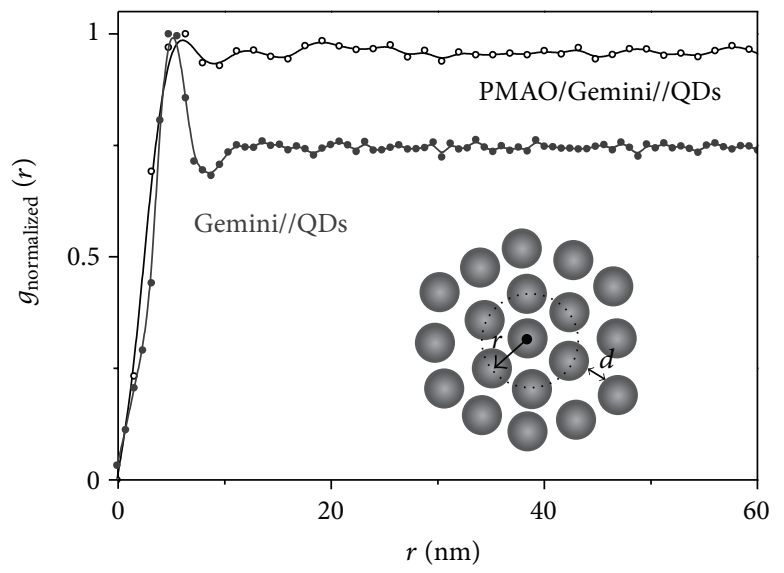

(e)

FIGURE 2: (a) 2D and 3D AFM images of Gemini surfactant LB films on mica. SEM (b) and TEM (c) images of Gemini//QDs LB films. The Gemini and QDs Langmuir monolayers were transferred at the surface pressure of 30 and $9 \mathrm{mN} \mathrm{m}^{-1}$, respectively. (d) RDF obtained from TEM images of QDs LB film onto Gemini and PMAO/Gemini LB films.

films to evaluate their stability [58] and to interpret the nanostructures formed by nanoparticles deposited onto different substrates [59]. Therefore, we analyzed the spreading parameter indicative of the balance between QDs and the coating layer (adhesive forces) and QD-QD (cohesive forces) intermolecular forces.
The spreading parameter, $S$, is calculated by $[18,58]$

$$
S=\gamma_{\text {layer 1/air }}-\left(\gamma_{\mathrm{QDs} / \text { layer } 1}+\gamma_{\mathrm{QDs} / \text { air }}\right),
$$

where $\gamma_{\text {layer 1/air }}, \gamma_{\mathrm{ODs} / \text { layer 1 }}$, and $\gamma_{\mathrm{QDs} / \text { air }}$, represent the surface tensions at the layer 1-air, QDs-layer 1, and QDs-air interfaces, 
TABLE 1: Spreading coefficients and interfacial tension values for the different systems studied.

\begin{tabular}{lccc}
\hline Bilayer systems & \multicolumn{2}{c}{$\begin{array}{c}\text { Interfacial tension } \\
\left(\mathrm{mN} \mathrm{m}^{-1}\right)^{\mathrm{c}}\end{array}$} & $\begin{array}{c}\text { Spreading coefficient } \\
\mathrm{S}\left(\mathrm{mN} \mathrm{m}^{-1}\right)\end{array}$ \\
\hline$\gamma_{\text {layer 1/air }}$ & $\gamma_{\mathrm{QD} / \text { layer 1 }}$ & \\
\hline PMAO//QDs & $33.7^{\mathrm{a}}$ & 0.0 & 0.7 \\
GMABEE//QDs & $39.3^{\mathrm{b}}$ & $8.3^{\mathrm{b}}$ & -2.0 \\
Gemini//QD & 36.0 & 0.0 & 3.0 \\
\hline
\end{tabular}

${ }^{\mathrm{a}}[51] ;{ }^{\mathrm{b}}[52] ;{ }^{\mathrm{c}}$ Layer 1 refers to the substrate coating film. See text.

respectively. The layer 1 notation refers to the substrate coating layer formed by polymers or surfactant molecules. The QDs/air surface tension value $\gamma_{\mathrm{QDs}}$ /air used in these calculations was taken from the literature as $33 \mathrm{mN} \mathrm{m}^{-1}$ [60]. The $\gamma_{\text {polymer/air }}$ for both polymers was approximated to the surface tensions of the hydrophobic moiety exposed to the air [31], linear polyethylene (PE) for the polymer PMAO [51], and polystyrene (PS) for PS-MA-BEE [52], respectively. Since the interface between PS-MA-BEE and QDs is constituted by styrene groups of the polymer PS-MA-BEE and hydrocarbon chains corresponding to the QDs ligand (TOPO), the $\gamma_{\text {PSMABEE/QDs }}$ value was taken as the interfacial tension between polystyrene and polyethylene molecules $\gamma_{\mathrm{PS} / \mathrm{PE}}=$ $8.3 \mathrm{mN} \mathrm{m}^{-1}[31,52]$. In the case of PMAO//QDs film, the contact between the polymer film and the QDs ligand is through the hyrocarbon chains, therefore the interfacial tension was considered as zero.

The Gemini/air surface tension, $\gamma_{\text {Gemini/air }}$, was calculated by combining the Young's equation and the Equation-ofState resulting in the following equation for the cosine of the contact angle, $\theta[61]$ :

$$
\begin{aligned}
\cos \theta= & -1+2 \sqrt{\gamma_{\text {Gemini/air }} / \gamma_{\text {water/air }}} \\
& \times \exp \left[-0.0001247\left(\gamma_{\text {water/air }}-\gamma_{\text {Gemini/air }}\right)^{2}\right] .
\end{aligned}
$$

To calculate the $\gamma_{\text {Gemini/air }}$ value, we take the contact angle as $80^{\circ}$ from the literature [20], and we consider $\gamma_{\text {Gemini/QDs }}$ as zero because the contact between the Gemini film and the QDs ligand (TOPO) is through hydrocarbon chains. In Table 1 the surface tension and spreading coefficient values calculated for the different systems. It is interesting to notice that the spreading parameter $S$ is negative for the PS-MABEE//QDs system. This fact means that QDs do not spread well on the PS-MA-BEE film, and the nanoparticles aggregate in islands, as shown in Figures 1(a) and 1(c). Conversely, the $S$ parameter corresponding to the Gemini//QDs system is the most positive one. This indicates that the QDs wet the surfactant film well, favoring the adsorption of nanoparticles on the surfactant film. As a consequence, the surfactant film induces a high QDs coverage. Since the surfactant forms an almost continuous and homogeneous film, the QDs are homogeneously distributed along it, as shown in Figure 2(d). An intermediate situation was observed for PMAO//QDs films. In this case the $S$ value is positive, indicating that the QDs wetting is good, but smaller than that is calculated for the surfactant, as shown in Table 1. In addition, the PMAO film used as substrate coating presents small holes. These two facts render a less efficient QDs adsorption on this film than onto the Gemini one.

In an attempt to improve the adsorption ability of the PMAO coating film, we explored mixtures of PMAO and Gemini surfactant. It is well established that polymer/surfactant mixed monolayers show synergistic effects in several film surface properties [62], such as the surface concentration. Therefore, we expect that mixed LB films could improve the QDs adsorption. Since the synergistic effects are usually related to attractive interactions between components at the interface, prior to the LB deposition we carried out the thermodynamic study of these mixtures by recording the surface pressure isotherms of mixed monolayers with different composition.

In Figure 3(a) some of these isotherms are represented. It is well established that the ideal behavior of a mixed monolayer can be related to the component miscibility, which is studied by means of the dependence of the excess area, $A_{\text {exc }}$, with the mixture composition. Therefore, we analyzed the variation of the excess area calculated from the isotherms and [63]: $A_{\text {exc }}=A_{12}-\left(X_{1} A_{1}+X_{2} A_{2}\right)$. In this equation $A_{12}$ represents the mean area per molecule in the mixed monolayer, and $A_{1}, A_{2}$ are the areas per molecule of the pure components at the same surface pressure value. Finally, $X_{1}$ and $X_{2}$ represent the mole fraction of each component at the interface. The excess area can be zero when the two components are immiscible or when the components form an ideal mixture, while the deviation from zero is signature of interactions between the components [63]. In Figure $3(\mathrm{~b})$ the $A_{\text {exc }}$ values are plotted against the Gemini mole fraction, $X_{\text {Gemini }}$. Negative deviations occur for all compositions and the most negative one appears around $X_{\text {Gemini }}=0.33$. This behavior indicates the existence of attractive interactions between molecules at the interface resulting in highly packed and elastic monolayers, as shown in Figures 3(a) and 3(c), respectively. Accordingly, we transferred a mixed PMAO/Gemini Langmuir monolayer of composition $X_{\text {Gemini }}=0.33$ from the air-water interface onto mica at the surface pressure value of $30 \mathrm{mN} \mathrm{m}^{-1}$. We selected this surface pressure because it corresponds to a dense state in which the monolayers do not reach the collapse. For the sake of comparison, the QDs monolayer was transferred at the same surface pressure employed to build the other QDs films, $9 \mathrm{mN} \mathrm{m}^{-1}$. The AFM image of the PMAO/Gemini $\left(X_{\text {Gemini }}=0.33\right)$ LB film without QDs is presented in Figures 4(a) and 4(b). The image shows an almost continuous film of PMAO and surfactant molecules with a height profile of $2 \mathrm{~nm}$. AFM and TEM images of PMAO/Gemini//QDs LB films are collected in Figures 4(c) and 4(d), respectively. These images show big QDs domains of similar morphology to those observed when the surfactant is used to cover the substrate. This indicates that the addition of small amounts of the Gemini surfactant to the PMAO monolayer leads to QDs films of similar structure to that observed on the Gemini film. To analyze possible differences between the 


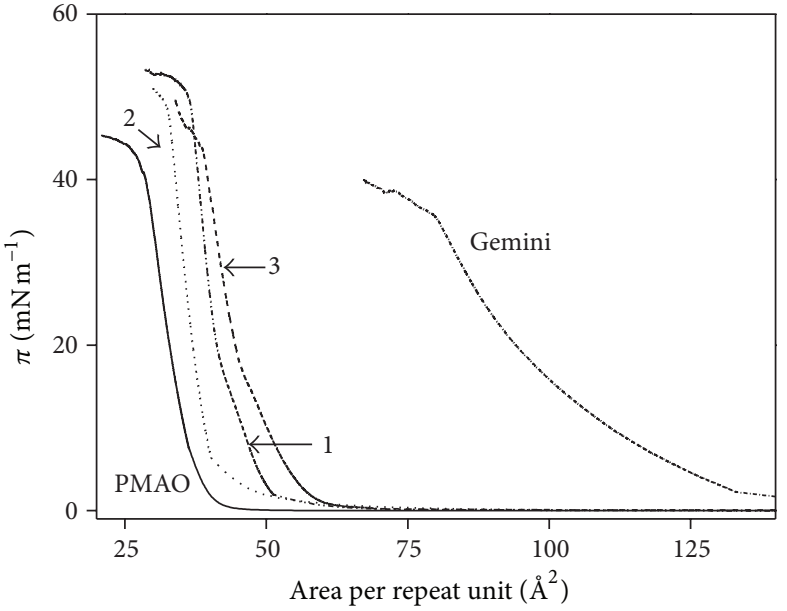

(a)

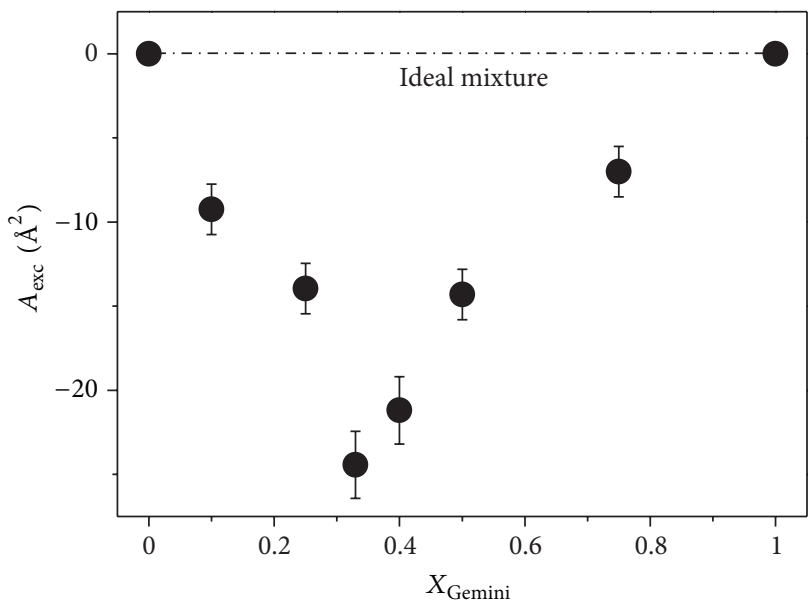

(b)

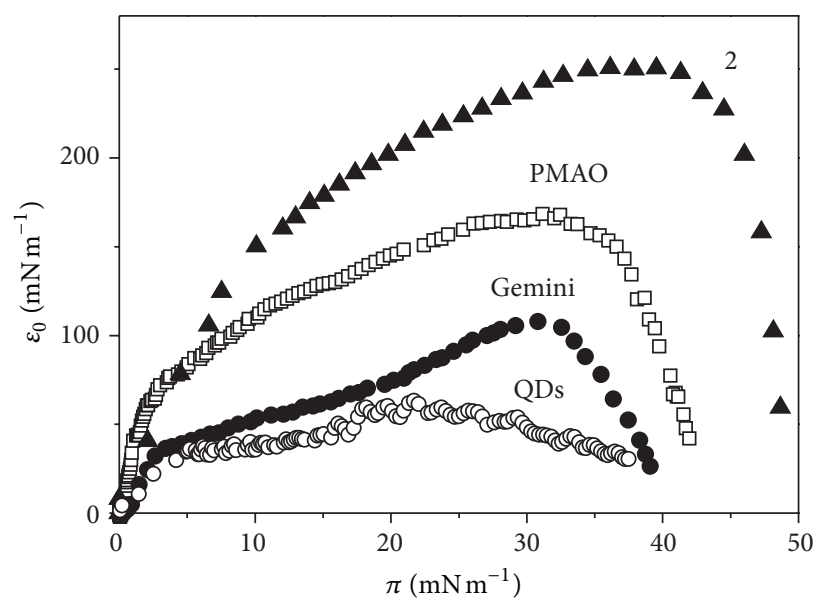

(c)

Figure 3: (a) Surface pressure isotherms of PMAO/Gemini mixed Langmuir monolayers at surfactant mole fraction of: 0.25 (1); 0.33 (2); 0.40 (3); PMAO and Gemini surface pressure isotherms are also represented. (b) Excess molecular area of mixing $\left(A_{\text {exc }}\right)$ versus composition for PMAO/Gemini mixed monolayers. (c) The equilibrium surface elasticity modulus versus surface pressure of QDs, PMAO, Gemini and PMAO/Gemini mixed $\left(X_{\text {Gemini }}=0.33\right)(2)$ Langmuir monolayers.

architecture of QDs films deposited onto the surfactant 18-218 and onto the PMAO/surfactant mixed films, we analyzed the RDF function obtained from the images of these films. For comparison, we represented the two radial distribution functions in Figure 2(e). In Figure 4(d), a representative TEM image corresponding to the areas selected for RDF analysis is presented. Results show that the first RDF peak for QDs deposited onto the PMAO/Gemini//QDs films is centered at $5.5 \pm 0.1 \mathrm{~nm}$, while the one corresponding to Gemini//QDs is centered at $5.1 \pm 0.1 \mathrm{~nm}$. To gain insights into the arrangement of QDs in the two LB films, we evaluated the number of particles in the successive shells $\left(N_{i} ; i=1,2, \ldots\right)$ from the integration of the RDF individual peaks. The first two shells contain ca. 5 and 10 particles for QDs deposited onto the Gemini LB film. This is compatible with an arrangement close to an ideal hexagonal array $\left(N_{1}=6, N_{2}=12\right)$. In contrast, to QDs films adsorbed on the PMAO/Gemini LB film, the number of particles in the first and second shell is 5 and 7 , respectively. This fact indicates that the hexagonal array of QDs adsorbed on the Gemini LB film is slightly distorted by the addition of PMAO at the Gemini surfactant coating film.

\section{Conclusions}

The results obtained in this work demonstrate that the morphology of the QDs films depends on the interfacial interactions between nanoparticles and the molecules employed to coat the solid substrate. The different morphologies were related to both the film wettability and the morphology of polymer and surfactant films used as substrate coatings. Thus the surfactant film showed the best wettability, and since it is constituted by close-packed surfactant molecules, 


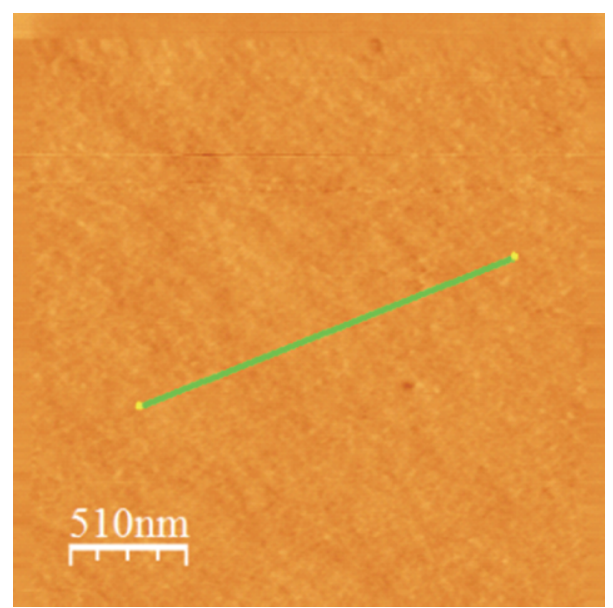

(a)
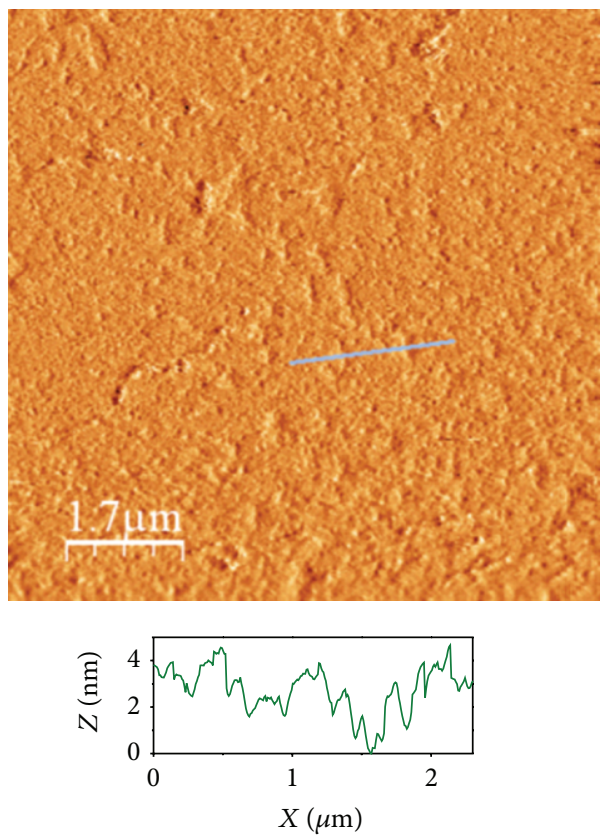

(c)
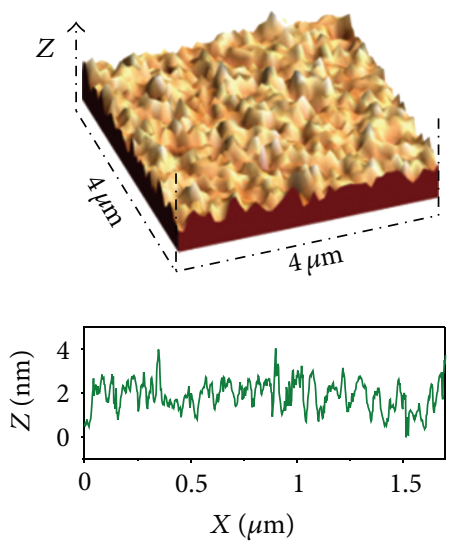

(b)

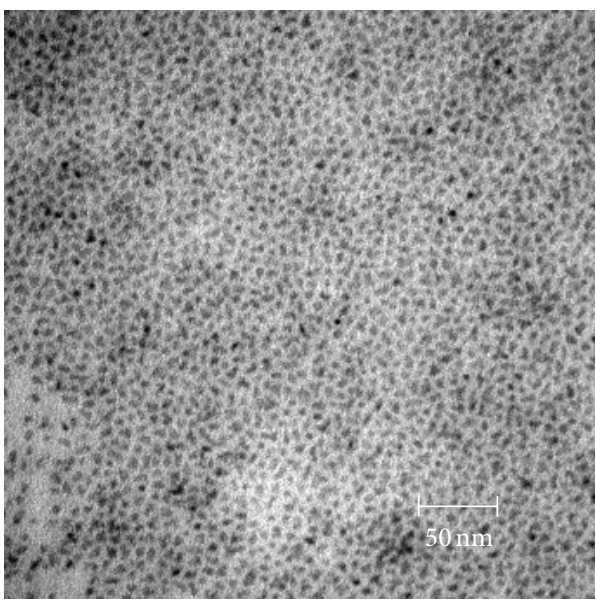

(d)

Figure 4: (a) 2D and 3D AFM images of PMAO/Gemini mixed LB films on mica. AFM (b) and TEM (c) images of PMAO/Gemini//QDs LB films. The PMAO/Gemini and QDs Langmuir monolayers were transferred at the surface pressure of 30 and $9 \mathrm{mN} \mathrm{m}^{-1}$, respectively. The $\mathrm{PMAO} / \mathrm{Gemini}$ mixture was prepared at the surfactant mole fraction of 0.33 .

this renders an almost continuous film of QDs. Conversely, when nanoparticles do not wet properly the film, the QDs aggregate in islands. This situation was observed for QDs deposited onto PS-MA-BEE films. An intermediate situation is observed for PMAO//QDs films. In this case, although the QDs wet the PMAO film well, the deposition is less efficient than on the surfactant. This fact is probably caused by the absence of covered polymer areas observed for PMAO films [45]. Finally, our results demonstrate that it is possible to modulate the QDs assembly on solids by modifying the nature of the molecules selected to coat the solid substrate.

\section{Acknowledgments}

The authors thank financial support from ERDF and MEC (MAT 2010-19727). T. Alejo and B. Martin-Garcia wish to thank the European Social Fund and Consejería de Educación de la Junta de Castilla y León for their FPI grants. The authors also thank to Dr. J. A. Pérez-Hernández and UltraIntense Lasers Pulsed Center of Salamanca (CLPU) for the AFM measurements, to Dr B. Rodríguez and International Iberian Nanotechnology Laboratory (INL) for the FIB/SEM facility, and the Electron Microscopy Service (Universidad de Salamanca) for the TEM measurements. 


\section{References}

[1] D. V. Talapin, J.-S. Lee, M. V. Kovalenko, and E. V. Shevchenko, "Prospects of colloidal nanocrystals for electronic and optoelectronic applications," Chemical Reviews, vol. 110, no. 1, pp. 389458, 2010

[2] A. P. Alivisatos, K. P. Johnsson, X. Peng et al., "Organization of 'nanocrystal molecules' using DNA," Nature, vol. 382, no. 6592, pp. 609-611, 1996.

[3] D. V. Talapin and C. B. Murray, "Applied physics: PbSe nanocrystal solids for $\mathrm{n}$ - and $\mathrm{p}$-channel thin film field-effect transistors," Science, vol. 310, no. 5745, pp. 86-89, 2005.

[4] T. P. Bigioni, X.-M. Lin, T. T. Nguyen, E. I. Corwin, T. A. Witten, and H. M. Jaeger, "Kinetically driven self assembly of highly ordered nanoparticle monolayers," Nature Materials, vol. 5, no. 4, pp. 265-270, 2006.

[5] J. L. Baker, A. Widmer-Cooper, M. F. Toney, P. L. Geissler, and A. P. Alivisatos, "Device-scale perpendicular alignment of colloidal nanorods," Nano Letters, vol. 10, no. 1, pp. 195-201, 2010.

[6] Y. Gao and Z. Tang, "Design and application of inorganic nanoparticle superstructures: current status and future challenges," Small, vol. 7, no. 15, pp. 2133-2146, 2011.

[7] R. P. Sear, S.-W. Chung, G. Markovich, W. M. Gelbart, and J. R. Heath, "Spontaneous patterning of quantum dots at the airwater interface," Physical Review E, vol. 59, no. 6, pp. R6255R6258, 1999.

[8] V. Ganesan, "Some issues in polymer nanocomposites: theoretical and modeling opportunities for polymer physics," Journal of Polymer Science B: Polymer Physics, vol. 46, no. 24, pp. 26662671, 2008.

[9] M. R. Bockstaller and E. L. Thomas, "Optical properties of polymer-based photonic nanocomposite materials," Journal of Physical Chemistry B, vol. 107, no. 37, pp. 10017-10024, 2003.

[10] G. M. Wallraff and W. D. Hinsberg, "Lithographic imaging techniques for the formation of nanoscopic features," Chemical Reviews, vol. 99, no. 7, pp. 1801-1821, 1999.

[11] T. Ito and S. Okazaki, "Pushing the limits of lithography," Nature, vol. 406, no. 6799, pp. 1027-1031, 2000.

[12] R. Garcia, R. V. Martinez, and J. Martinez, "Nano-chemistry and scanning probe nanolithographies," Chemical Society Reviews, vol. 35, no. 1, pp. 29-38, 2006.

[13] G. Decher, "Fuzzy nanoassemblies: toward layered polymeric multicomposites," Science, vol. 277, no. 5330, pp. 1232-1237, 1997.

[14] T. Cui, F. Hua, and Y. Lvov, "Lithographic approach to pattern multiple nanoparticle thin films prepared by layer-by-layer selfassembly for microsystems," Sensors and Actuators A, vol. 114, no. 2-3, pp. 501-504, 2004.

[15] M. C. Petty, Langmuir-Blodgett Films: An Introduction, Cambridge University Press, Cambridge, UK, 1996.

[16] G. Zhavnerko and G. Marletta, "Developing Langmuir-Blodgett strategies towards practical devices," Materials Science and Engineering B, vol. 169, no. 1-3, pp. 43-48, 2010.

[17] K. Lambert, R. K. Čapek, M. I. Bodnarchuk et al., "Langmuirschaefer deposition of quantum dot multilayers," Langmuir, vol. 26, no. 11, pp. 7732-7736, 2010.

[18] D. Gentili, G. Foschi, F. Valle, M. Cavallini, and F. Biscarini, "Applications of dewetting in micro and nanotechnology," Chemical Society Reviews, vol. 41, no. 12, pp. 4430-4443, 2012.

[19] N. Tomczak, D. Jańczewski, M. Han, and G. J. Vancso, "Designer polymer-quantum dot architectures," Progress in Polymer Science, vol. 34, no. 5, pp. 393-430, 2009.
[20] M. Lv, Q. Chen, M. Yonese, S. Xu, and H. Liu, "Auto-organized nano-structure of collagen on Gemini surfactant monolayer," Colloids and Surfaces B: Biointerfaces, vol. 61, no. 2, pp. 282-289, 2008.

[21] V. Torrisi, F. Ruffino, A. Licciardello, M. G. Grimaldi, and G. Marletta, "Memory effects in annealed hybrid gold nanoparticles/block copolymer bilayers," Nanoscale Research Letters, vol. 6, no. 1, article 167, 2011.

[22] A. Sharma, C. M. Pandey, Z. Matharu et al., "Nanopatterned cadmium selenide Langmuir-Blodgett platform for leukemia Detection," Analytical Chemistry, vol. 84, no. 7, pp. 3082-3089, 2012.

[23] T.-H. Kim, K.-S. Cho, E. K. Lee et al., "Full-colour quantum dot displays fabricated by transfer printing," Nature Photonics, vol. 5, no. 3, pp. 176-182, 2011.

[24] X. Ji, C. Wang, J. Xu, J. Zheng, K. M. Gattás-Asfura, and R. M. Leblanc, "Surface chemistry studies of (CdSe)ZnS quantum dots at the air-water interface," Langmuir, vol. 21, no. 12, pp. 5377-5382, 2005.

[25] L. M. Bronstein, E. V. Shtykova, A. Malyutin et al., "Hydrophilization of magnetic nanoparticles with modified alternating copolymers. Part 1: the influence of the grafting," Journal of Physical Chemistry C, vol. 114, no. 50, pp. 21900-21907, 2010.

[26] E. V. Shtykova, X. Huang, X. Gao et al., "Hydrophilic monodisperse magnetic nanoparticles protected by an amphiphilic alternating copolymer," Journal of Physical Chemistry C, vol. 112, no. 43, pp. 16809-16817, 2008.

[27] W. W. Yu, E. Chang, C. M. Sayes, R. Drezek, and V. L. Colvin, "Aqueous dispersion of monodisperse magnetic iron oxide nanocrystals through phase transfer," Nanotechnology, vol. 17, no. 17, pp. 4483-4487, 2006.

[28] T. Pellegrino, L. Manna, S. Kudera et al., "Hydrophobic nanocrystals coated with an amphiphilic polymer shell: a general route to water soluble nanocrystals," Nano Letters, vol. 4, no. 4, pp. 703-707, 2004.

[29] J. Jin, L. S. Li, Y. Q. Tian et al., "Structure and characterization of surfactant-capped CdS nanoparticle films by the LangmuirBlodgett technique," Thin Solid Films, vol. 327-329, no. 1-2, pp. 559-562, 1998.

[30] A. Gole, N. R. Jana, S. T. Selvan, and J. Y. Ying, "LangmuirBlodgett thin films of quantum dots: synthesis, surface modification, and fluorescence resonance energy transfer (FRET) studies," Langmuir, vol. 24, no. 15, pp. 8181-8186, 2008.

[31] R. W. Zehner, W. A. Lopes, T. L. Morkved, H. Jaeger, and L. R. Sita, "Selective decoration of a phase-separated Diblock copolymer with thiol-passivated gold nanocrystals," Langmuir, vol. 14, no. 2, pp. 241-244, 1998.

[32] W. A. Lopes and H. M. Jaeger, "Hierarchical self-assembly of metal nanostructures on diblock copolymer scaffolds," Nature, vol. 414, no. 6865, pp. 735-738, 2001.

[33] R. S. Krishnan, M. E. Mackay, P. M. Duxbury et al., "Self-assembled multilayers of nanocomponents," Nano Letters, vol. 7, no. 2, pp. 484-489, 2007.

[34] R. Jones, C. S. Winter, R. H. Tredgold, P. Hodge, and A. Hoorfar, "Electron-beam resists from Langmuir-Blodgett films of poly(styrene/maleic anhydride) derivatives," Polymer, vol. 28, no. 10, pp. 1619-1626, 1987.

[35] S. John Collins, N. L. Mary, G. Radhakrishnan, and A. Dhathathreyan, "Studies of spread monolayers of derivative of styrene-maleic anhydride copolymers," Journal of the Chemical Society-Faraday Transactions, vol. 93, no. 22, pp. 4021-4023, 1997. 
[36] Y. Tian and J. H. Fendler, "Langmuir-Blodgett film formation from fluorescence-activated, surfactant-capped, size-selected CdS nanoparticles spread on water surfaces," Chemistry of Materials, vol. 8, no. 4, pp. 969-974, 1996.

[37] Y.-J. Shen, Y.-L. Lee, and Y.-M. Yang, "Monolayer behavior and Langmuir-Blodgett manipulation of CdS quantum dots," Journal of Physical Chemistry B, vol. 110, no. 19, pp. 9556-9564, 2006.

[38] K. M. Gattás-Asfura, C. A. Constantine, M. J. Lynn, D. A. Thimann, X. Ji, and R. M. Leblanc, "Characterization and 2D selfassembly of CdSe quantum dots at the air-water interface," Journal of the American Chemical Society, vol. 127, no. 42, pp. 14640-14646, 2005.

[39] R. Zana, "Dimeric and oligomeric surfactants. Behavior at interfaces and in aqueous solution: a review," Advances in Colloid and Interface Science, vol. 97, no. 1-3, pp. 205-253, 2002.

[40] A. Singh, J. D. van Hamme, and O. P. Ward, "Surfactants in microbiology and biotechnology: part 2. Application aspects," Biotechnology Advances, vol. 25, no. 1, pp. 99-121, 2007.

[41] X. Chen, J. Wang, N. Shen et al., "Gemini surfactant/DNA complex monolayers at the air-water interface: effect of surfactant structure on the assembly, stability, and topography of monolayers," Langmuir, vol. 18, no. 16, pp. 6222-6228, 2002.

[42] Q. Chen, X. Kang, R. Li et al., "Structure of the complex monolayer of gemini surfactant and DNA at the air/water Interface," Langmuir, vol. 28, no. 7, pp. 3429-3438, 2012.

[43] D. López-Díaz and M. M. Velázqueza, "Evidence of glass transition in thin films of maleic anhydride derivatives: effect of the surfactant coadsorption," European Physical Journal E, vol. 26, no. 4, pp. 417-425, 2008.

[44] S. Gupta, N. Singh, M. Sastry, R. Kakkar, and R. Pasricha, "Controlling the assembly of hydrophobized gold nanoparticles at the air-water interface by varying the interfacial tension," Thin Solid Films, vol. 519, no. 3, pp. 1072-1077, 2010.

[45] T. Alejo, M. D. Merchán, M. M. Velázquez, and J. A. PérezHernández, "Polymer/surfactant assisted self-assembly of nanoparticles into Langmuir-Blodgett films," Materials Chemistry and Physics, vol. 138, no. 1, pp. 286-294, 2013.

[46] P. K. Paul, S. A. Hussain, D. Bhattacharjee, and M. Pal, "Adsorption of cationic laser dye onto polymer/surfactant complex film," Chinese Journal of Chemical Physics, vol. 24, no. 3, pp. 348352, 2011.

[47] R. Zana, M. Benrraou, and R. Rueff, "Alkanediyl- $\alpha, \omega$-bis(dimethylalkylammonium bromide) surfactants. 1. Effect of the spacer chain length on the critical micelle concentration and micelle ionization degree," Langmuir, vol. 7, no. 6, pp. 1072-1075, 1991.

[48] T. Alejo, M. D. Merchán, and M. M. Velázquez, "Specific ion effects on the properties of cationic Gemini surfactant monolayers," Thin Solid Films, vol. 519, no. 16, pp. 5689-5695, 2011.

[49] W. W. Yu and X. Peng, "Formation of high-quality CdS and other II-VI semiconductor nanocrystals in noncoordinating solvents: tunable reactivity of monomers," Angewandte Chemie International Edition, vol. 41, no. 13, pp. 2368-2371, 2002.

[50] J. Jasieniak, L. Smith, J. van Embden, P. Mulvaney, and M. Califano, "Re-examination of the size-dependent absorption properties of CdSe quantum dots," Journal of Physical Chemistry C, vol. 113, no. 45, pp. 19468-19474, 2009.

[51] R. H. Dettre and R. E. Johnson Jr., "Surface properties of polymers. I. The surface tensions of some molten polyethylenes," Journal of Colloid And Interface Science, vol. 21, no. 4, pp. 367377, 1966.
[52] J. E. Mark, Polymer Data Handbook, Oxford University Press, New York, NY, USA, 1999.

[53] B. Martín-García, M. M. Veláquez, J. A. Pérez-Hernández, and J. Hernández-Toro, "Langmuir and Langmuir-Blodgett films of a maleic anhydride derivative: effect of subphase divalent cations," Langmuir, vol. 26, no. 18, pp. 14556-14562, 2010.

[54] B. Martín-García and M. M. Velázquez, "Block copolymer assisted self-assembly of nanoparticles into Langmuir-Blodgett films: effect of polymer concentration," Materials Chemistry and Physics, 2013.

[55] K. Holmer, B. Jönsson, B. Kronberg, and B. Lindman, Surfactants and Polymers in Aqueous Solution, John Wiley \& Sons, New York, NY, USA, 2003.

[56] ImageJ Software.

[57] J. Jiang, T. D. Krauss, and L. E. Brus, "Electrostatic force microscopy characterization of trioctylphosphine oxide selfassembled monolayers on graphite," Journal of Physical Chemistry B, vol. 104, no. 50, pp. 11936-11941, 2000.

[58] T. Baumgart and A. Offenhäuser, "Polysaccharide-supported planar bilayer lipid model membranes," Langmuir, vol. 19, no. 5, pp. 1730-1737, 2003.

[59] S. Kundu and J. K. Bal, "Reorganization of Au nanoparticle Langmuir-Blodgett films on wet chemically passivated $\mathrm{Si}(001)$ surfaces," Journal of Applied Physics, vol. 110, no. 11, Article ID 114302, 2011.

[60] Y. Lin, A. Böker, J. He et al., "Self-directed self-assembly of nanoparticle/copolymer mixtures," Nature, vol. 434, no. 7029, pp. 5559, 2005.

[61] J. K. G. Bunk, D. U. Pospiech, K.-J. Eichhorn et al., "Studying the influence of chemical structure on the surface properties of polymer films," Colloids and Surfaces A, vol. 362, no. 1-3, pp. 4757, 2010.

[62] J. T. Davies and E. K. Rideal, Interfacial Phenomena, Academic Press, New York, NY, USA, 1963.

[63] G. L. Gaines, Insoluble Monolayers at the Air-Water Interface, Interscience Publishers, Hoboken, NJ, USA, 1966. 

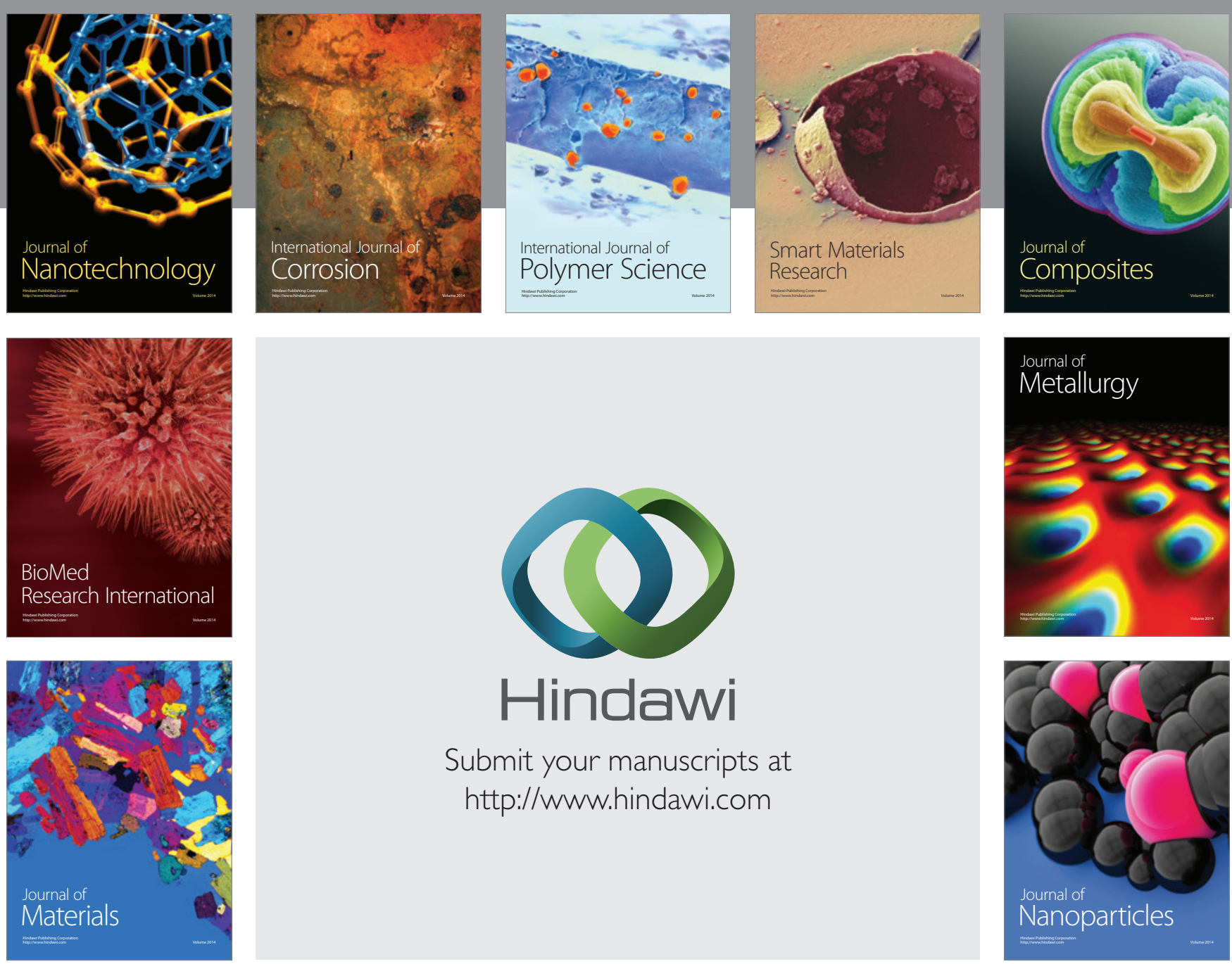

Submit your manuscripts at http://www.hindawi.com
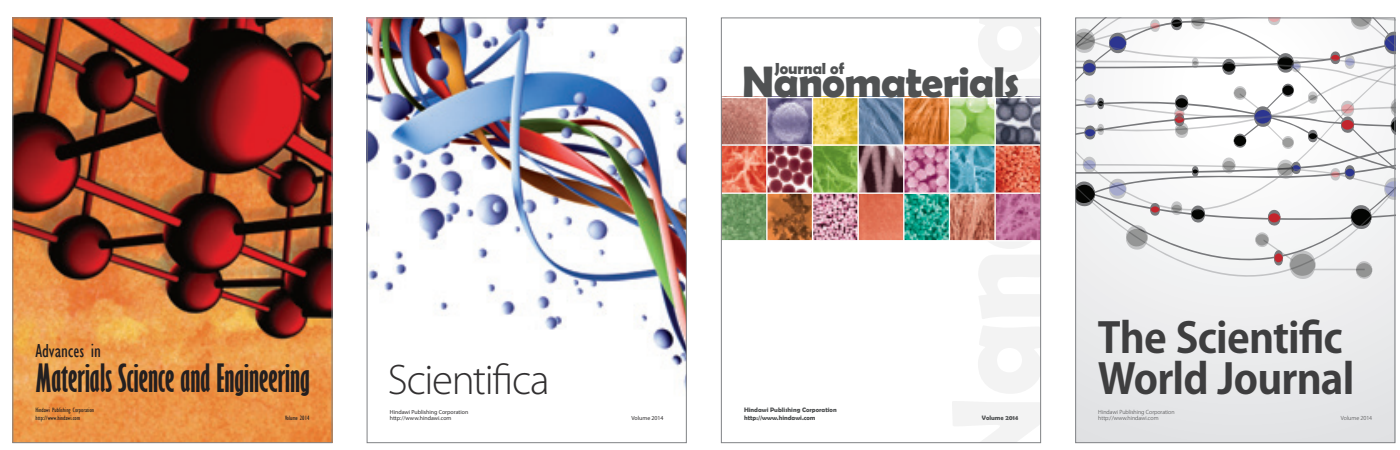

\section{The Scientific World Journal}
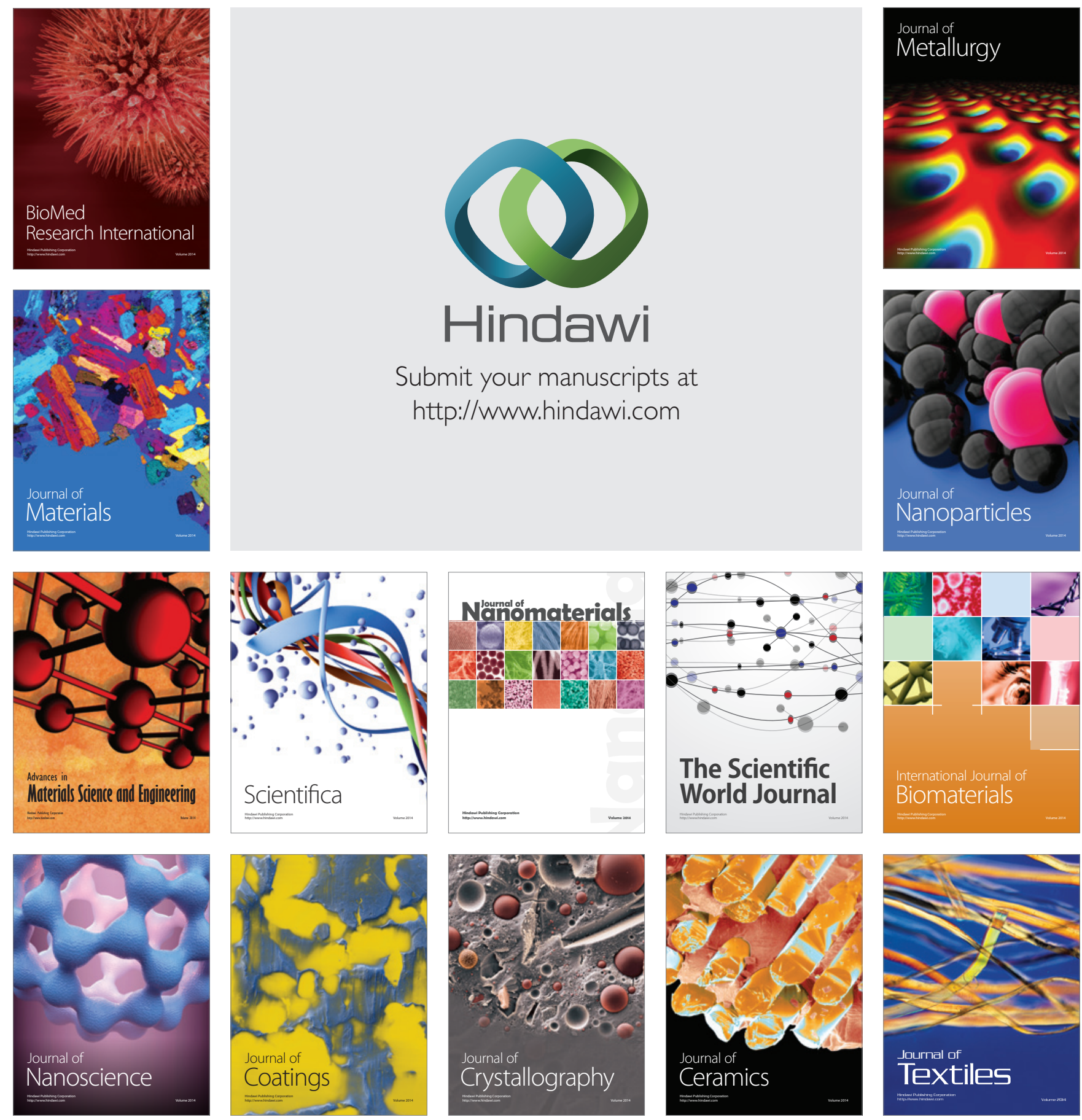